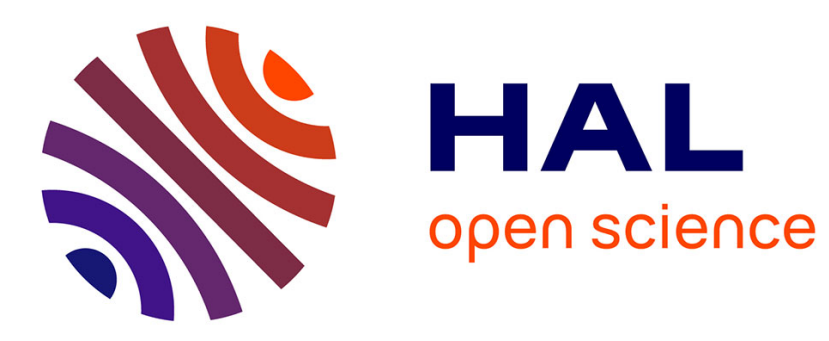

\title{
From 3D Bimanual Toward Distant Collaborative Interaction Techniques: An Awareness Issue
}

Morgan Le Chénéchal, Bruno Arnaldi, Thierry Duval, Valérie Gouranton, Jérôme Royan

\section{To cite this version:}

Morgan Le Chénéchal, Bruno Arnaldi, Thierry Duval, Valérie Gouranton, Jérôme Royan. From 3D Bimanual Toward Distant Collaborative Interaction Techniques: An Awareness Issue. 2014 International Workshop on Collaborative Virtual Environments (3DCVE), IEEE VR, Mar 2014, Minneapolis, United States. 10.1109/3DCVE.2014.7160929 . hal-00974018

\section{HAL Id: hal-00974018 https://hal.inria.fr/hal-00974018}

Submitted on 4 Apr 2014

HAL is a multi-disciplinary open access archive for the deposit and dissemination of scientific research documents, whether they are published or not. The documents may come from teaching and research institutions in France or abroad, or from public or private research centers.
L'archive ouverte pluridisciplinaire HAL, est destinée au dépôt et à la diffusion de documents scientifiques de niveau recherche, publiés ou non, émanant des établissements d'enseignement et de recherche français ou étrangers, des laboratoires publics ou privés. 


\section{From 3D Bimanual Toward Distant Collaborative Interaction Techniques: An Awareness Issue}

\author{
Morgan Le Chénéchal* \\ IRT $\mathrm{b}<>$ com
}

\author{
Bruno Arnaldi ${ }^{\dagger}$ \\ INSA of Rennes \\ IRISA/INRIA \\ IRT $\mathrm{b}<>$ com
}

\author{
Thierry Duval ${ }^{\dagger}$ \\ University of Rennes 1 \\ IRISA/INRIA \\ IRT $\mathrm{b}<>$ com
}

\author{
Valérie Gouranton \\ INSA of Rennes \\ IRISA/INRIA \\ IRT $\mathrm{b}<>$ com
}

\author{
Jérôme Royan* \\ IRT $b<>$ com
}
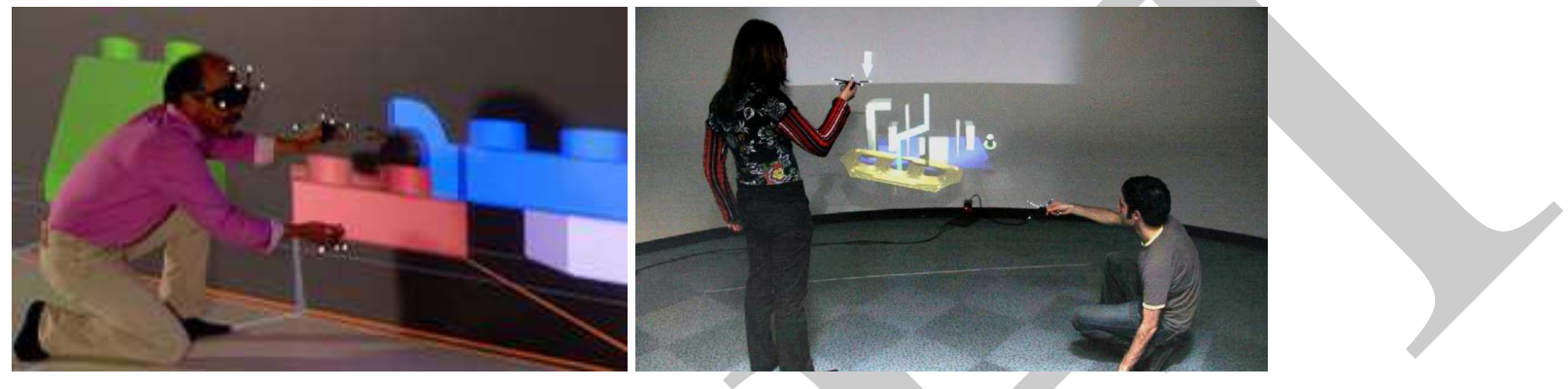

Figure 1: These two figures state the question: How close are 3D bimanual interactions to collaborative interactions?

\begin{abstract}
This paper aims to raise the question : "How much 3D bimanual interaction techniques can be useful to the design of collaborative interaction techniques in the field of Collaborative Virtual Environment (CVE)?". Indeed, CVE involve the use of complex interaction techniques based on specific collaborative metaphors. The design of these metaphors may be a difficult task because it has to deal with collaborative issues that came from sparse research areas (Human-Computer Interfaces, Human-Human Interactions, Networking, Physiology and Social Psychology). Metaphors for bimanual interactions have been developed for a while essentially because it is a widely spread area of interest for common tasks. Bimanual interactions involve the simultaneous use of both hands of the user in order to achieve a goal with better performances compared to uni-manual interactions thanks to a natural skill that is proprioception. This collaborative aspect could certainly be a helpful entry point in the design of efficient collaborative interaction techniques extended from improved bimanual metaphors. However, the proprioceptive sense cannot be considered in the same way, and additional features must be proposed to be able to collaborate efficiently. Thus, awareness is a key to let CVE be usable and the availability of collaborative feedbacks is essential to extend bimanual interactions toward collaborative ones. In this paper, we based our study on existing work on bimanual and collaborative interaction techniques trying to draw similarities between them. We emphasize common points between both fields that could be useful to better design both metaphors and awareness in CVE.
\end{abstract}

Index Terms: A.1 [Introductory and Survey]; H.5.3 [Information Interfaces and Presentations]: Group and Organization InterfacesComputer-supported cooperative work; I.3.6 [Computer Graphics]: Methodology and Techniques - Interaction techniques; I.3.7 [Computer Graphics]: Three-Dimensional Graphics and RealismVirtual reality;

\footnotetext{
* \{morgan.lechenechal,jerome.royan $\} @ b$-com.com

$\dagger\{$ bruno.arnaldi,thierry.duval,valerie.gouranton\} @ irisa.fr
}

\section{INTRODUCTION}

Human-Computer Interfaces (HCI) have often taken advantage of the natural bimanual skills of humans. In the late of eighty's, pioneers as Zimmerman imagined advanced interfaces such as the Data Glove ${ }^{\mathrm{TM}}[52]$ enabling natural gestures of the hands as interface. This kind of interface paved the way to first work about natural bimanual interactions for VR. It took place fifteen years ago and proposed a set of 3D manipulation techniques [13] as intuitive and immersive as possible. HCI using both hands rather than a single one provide interaction techniques closer to the real ones and can increase the accuracy of the interactions. These research work on bimanual interactions are strongly based on natural skills of humans including the proprioceptive sense as an essential part. Thus, some work proposed asymmetric interactions [29] (e.g considering a different role for the dominant hand and the non-dominant one) inspired by physiological results and proved that it is an efficient and natural way to interact using both hands. In this paper, we restrict bimanual interactions to this kind of systems, using free motion and gestures of the hands to interact.

Another way to improve performances is to provide multi-user systems in order to cross the skills of each collaborators. Computersupported cooperative work (CSCW) is a powerful tool to help people to collaborate in a distant and efficient way. Distant users can share some virtual contents to collaborate, analyze and act on and with them in real-time. CSCW systems aim to virtually reduce the real distance between collaborators by sharing a common virtual space. For instance, users can experiment teleconferencing experiences as if they were in the same room [6] or share 3D models in a common virtual environment (VE) [42]. Users are not always collaborating in a remote way: they can also collaborate locally, which obviously simplify the understanding of the other's actions (e.g face-to-face setup [35] or co-located setup [46]). But when distant users are collaborating, specific feedbacks must be provided to let users be aware of the other participants, their actions, their focus and their interaction capabilities. These types of feedback, as well as every available feedbacks about user's actions on the system, are encapsulated in the term awareness.

Virtual reality (VR) systems are widely used in conception, training or simulation and provide useful feedback in immersive setups. In this context, these feedbacks are essential because the user is 
immersed in a virtual world and isolated from the real world in order to have a sensory-motor and cognitive activity in an interactive virtual world [23]. Research on CSCW and VR converge in the field of collaborative virtual environment (CVE). Indeed, CVE have focused in both interaction techniques and awareness provided in CSCW and VR applications. Thus, CVE propose shared virtual environments to perform immersive virtual tasks such as scientific data analysis [19] or training tasks [33] in a natural way. CVE aim to improve performances in many ways, such as usability, task completion time or environment perceptions. But CVE require adequate interaction techniques to support collaborative interactions and specific metaphors for concurrent interactions handling as well as awareness features.

In this survey, we try to analyze both fields, 3D bimanual and collaborative interaction techniques, and we aim to draw a parallel between some of these techniques as extensions from bimanual toward collaborative interactions. We will address the main issue to extend bimanual toward distant collaborative interactions that is the lack of awareness. Section 2 presents an overview of both bimanual and CVE applications motivating our analysis. Then, section 3 classifies the interactions according to the main interactive features. Section 4 presents the principles used to enhance interaction performances proposed by the literature in bimanual setups. Section 5 explains the need for CVE to overcome the limitations of bimanual interactions developing collaborative interaction techniques and the involving awareness issues that will be addressed in section $6 \mathrm{Fi}$ nally, section 7 concludes this paper.

\section{Overview \& Motivations}

Let us consider two users in collaborative interactions, if each user interacts with only one hand, we state that it can be compared to bimanual interactions that can be considered as a collaboration between both hands 1 Indeed, bimanual interactions handle two different inputs of the same user that could be, in some cases, one input per user for two different users. Figure 1 illustrates this concept. This parallel is even more obvious if considering tabletop/surfacebased interactions (e.g tactile interactions) handling several inputs regardless of the number of users (locally [9] or remotely [12]). Thus, bimanual interactions can be a great entry point to understand key issues about collaboration. Several work have been done in bimanual VR applications with advanced features about 3D interaction techniques and awareness. Some of these features can certainly be useful in CVE and extended to handle more than two inputs with more than one user. This will lead to propose enhanced awareness features for collaboration.

First, subsection 2.1 focuses on bimanual requirements, then subsection 2.2 lists all CVE requirements. Last, subsection 2.3 highlights similarities between collaborative and bimanual interactions, and subsection 2.4 sums up the section.

\subsection{Bimanual requirements}

Basically, systems providing bimanual interactions must consider both hands of the user to control a single or a couple of hardware interfaces. In our study, we only consider bimanual interactions managed through two 6DOF tracking of the hands. We are not interested about static or 2D interfaces because they do not allow the use of advanced 3D interaction techniques based on proprioception.

In real bimanual interactions, people act with little cognitive effort [13], almost seamlessly with a millimeter precision [29]. Ideally, this accuracy and simple cognitive task aspect must also be obtained in bimanual interactive applications. To achieve it, physiological studies were performed in bimanual interactions and revealed the significant part of the kinaesthetic feedback and the

${ }^{1}$ With some limitations such as the use of two dominant hands in the collaborative case. proprioceptive sense when interacting collaboratively with both hands [4]. The cognitive load can also be influenced by the switching between modes of interaction (uni-manual vs. bimanual [13]).

Moreover, as any interactive system, bimanual systems must handle interactive features such as responsiveness and awareness. However, dealing with two independent inputs rather than a single one involves more difficulties. The user must be aware of the individual action of both hands in the system, as well as the common action applied by the merging of the two inputs. Thus, enhanced interactions (enabling infeasible tasks otherwise) compared with a uni-manual setup can be proposed, and the system must consider them and let the user to be aware of these additional interaction capabilities.

\subsection{CVE requirements}

To begin, we can paraphrase Joslin et al. [30] saying that " $C V E$ is VR plus lots of features". Indeed, it aims to propose VR applications for several users acting thanks to collaborative interactions. Users can be distant, thus a network layer has to handle consistency between the different sites. Moreover, it has to provide multi-modal communication and has to handle the virtual and real spaces of the heterogeneous users.

The deep analysis of networking issues is not the purpose of this paper, a good survey can be found in [18]. The network layer is directly developed from networking research results and we can consider that it provides us a reliable framework. It deals with shared objects over the network that can be dynamically modified by different users. Consequently it has to handle concurrency access, as well as latency issues due to the real-time constraint. Object distribution paradigms, such as the mirror/reference distribution paradigm, have been proposed. They adapt dynamically the distribution of objects on the network to enhance local interaction and distant collaboration. Indeed, CVE have to handle access rights policy, and choose on which site the behavior of an object is executed. This choice is influenced by network performances and current interactions. The network latency involves inconsistencies between distant sites in co-dependent interaction phase, and the distributed system must minimize the impact of inconsistency to not disturb remote co-dependent interactions.

The other features are related to interaction techniques. The multi-user aspect involves the need for specific interaction techniques, such as a 3-hand manipulation technique [3] to be used by two or three users. Moreover, if the system is aware of the real users space (and of their hardware setup), it can be represented in the VE in order to make the other users perceive it [15]. Thus, VE handle virtual spaces [47] to help the users to have a better mental representation of the whole $3 \mathrm{D}$ environment including the remote users.

Last, the remote aspect involves the need for communication between the different users. The multi-modality of the communication is important to enhance users' performances [38]. It helps synchronization between distant interactions and provides some kind of awareness. The audio channel is a primordial one, but other techniques can be employed such as the use of symbols that users can put in the VE [38] or even realistic [6] and emotive [21] avatars for non-verbal communication.

\subsection{Shared involvements}

First, bimanual as well as collaboration enhances interaction performances in many tasks too complex to be performed using a single input [36]. For example, Latulipe et al. [31] proposed a system to draw complex spline-curves increasing performances using users' both hands in a symmetric way. In CVE, interaction performances can be increased in many ways, such as providing multiple viewpoints available by the different users in a 3D selection task. 
Another similarity is the availability of multiple input-based interactions to achieve infeasible task in uni-manual setting. For instance the rotation of a cubic prop in [22] can be done only using both hands to hold it in a different orientation. In CVE, collaborators can act in a complementary way for example in finding objects in a complex VE [38].

With regard to these multiple input-based interaction techniques, several bimanual metaphors can be extended for collaboration, especially for object 3D manipulation. Indeed, some metaphors enabling a user to control an object manipulated with both hands are based on an asymmetric pattern where each hand controls a set of degrees of freedom (DOF) of that object. This principle of splitting DOF can consistently be reused for collaboration letting each user control only a subset of the DOF.

Last, Cutler et al. [13] raise the issue about the transitions between uni-manual and bimanual interactions. These transitions must not disturb the user and can be defined as explicit or implicit ones according to the context. They have to be seamless and natural. The same issue is encountered in CVE where transitions between mono and multi-user interactions have to be as natural as possible [41].

\subsection{Summary}

In this section we presented an overview of both fields we are interested in: bimanual interactions and CVE. We developed all requirements to build a CVE and explain why it is a complex task. Last, we highlighted some similarities encountered in both fields. These similarities induce same kinds of issues when developing bimanual or collaborative interaction techniques. Thus, both fields can be studied in parallel in order to capitalize on common solutions proposed in each fields.

\section{INTERACTION CLASSIFICATION}

The literature proposes classifications for bimanual interactions as well as for collaborative ones. These classifications are presented respectively in subsection 3.1 and subsection 3.2 then a comparative discussion is provided in subsection 3.3

\subsection{Bimanual classification}

Bimanual interactions can be split into two main classes [29]:

- symmetric interactions,

- asymmetric interactions.

First, symmetric interactions do not make distinction between the role of both hands. For example, "bring a ball" [25] or the two-hand "SkeweR" technique [14] are symmetric interactions.

Second, asymmetric interactions differentiate the role of each hand. In this case, the dominant hand is the skillful hand (the right hand for right-handed people) that can perform fine grain interactions, while the non-dominant hand is used for coarser interactions. Asymmetric bimanual interaction has been deeply studied by Guiard [28] and its principles are detailed in subsection 4.1 .

\subsection{Collaboration classification}

In the literature several authors classified collaborative interactions. We present our classification based on a mix between the ones presented in [34, 33]. It is a hierarchical classification from the basic features to the more advanced ones.

- Level 1: Awareness of team members' presence (avatars and communication channels).

- Level 2: Individual interactions on the scene.

- Level 3: Collaborative/codependent interactions (at the same time).
- Level 3.1: Modification of different attributes.

- Level 3.2: Modification of the same attribute.

Level 2 is not restrained to collaboration because it is equivalent to a single user interaction in a VE. However, it is useful to explicit it because some collaborative applications only provide this level of interaction (and the Level 1) and do not handle codependent interaction (Level 3). Level 3 is the more advanced feature offered by $\mathrm{CVE}$, and it involves complex issues linked firstly to the handling of multiple inputs and secondly to the network latency and/or failures.

\subsection{Comparative discussion}

In some bimanual manipulation tasks as the "bring a ball" presented in [25], the interaction is based on a symmetric collaboration between both hands. Level 3.2 is the collaborative equivalence of this bimanual interaction paradigm. The same way, Level 3.1 is based on a partial DOF control by each user that is used in most of the bimanual manipulation tasks based on asymmetric interaction.

Thus, these classifications are a starting point of a comparison between bimanual and collaborative interactions. We develop this idea of comparable interaction paradigm further in the next section, especially in subsection 5.2

\section{Bimanual interactions}

Improved performances in bimanual interactions are not only due to the use of additional inputs into the system, but also to the user proprioceptive sense. A human always knows where his both hands are relatively to each other and to his body. This natural proprioceptive information provides essential cues to be able to act seamlessly without looking at his hands all the time. Exploiting proprioception and the different skill capabilities of each hand leads to propose enhanced interaction techniques. First, subsection 4.1 presents bimanual interactions based on an asymmetric use of each hand, then subsection 4.2 provides some counter-examples based on symmetric interactions.

\subsection{Asymmetric paradigm: bimanual interactions based on a frame of reference}

Bimanual interaction has been well-studied by physiological researchers. HCI community used this research to design better their interaction techniques according to important findings about the asymmetry of human bimanual gestures. In [13], Cutler et al. use a right-handed writer as an example to summarize the following main principles:

- Right-to-left reference: the writing right hand moves according to the left hand motion that holds the paper.

- Asymmetrical scales of motion: the right hand motion is much more accurate than the left hand motion (writing task vs. move the paper).

- Left-hand precedence: the left hand begins positioning paper, then the right hand starts to write.

These principles have been used for various interactions such as selection task [50] or 3D manipulation [29]. This last work by Hinckley et al. perfectly illustrates interactions based on a dynamic frame of reference. The user brings two props in each hands, the non-dominant one representing the world-frame of reference in their context, and the dominant one controlling an object moving in the VE. In their surgical application, the moving object must be precisely placed into the 3D VE. Better performances are obtained with the participation of the left hand as a frame of reference. Accuracy is enhanced thanks to proprioception compared with the same uni-manual task. 


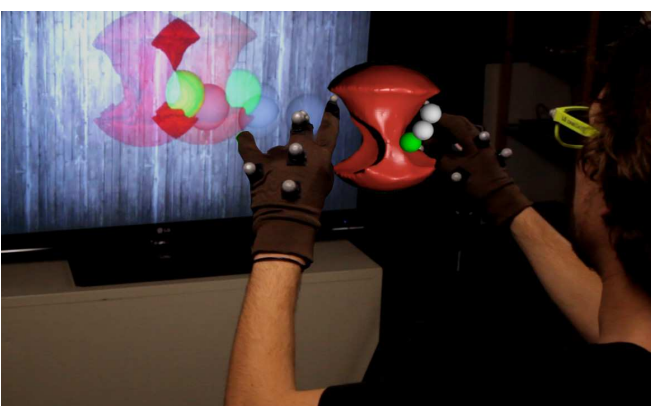

Figure 2: Illustration of a virtual design immersive system based on natural bimanual interactions proposed in [32]

\subsection{Counter-examples: bimanual symmetric interac- tions}

According to bimanual interaction principles proposed by Guiard [28], bimanual interactions are intrinsically asymmetric. However, some tasks proved to be more usable with symmetric bimanual interactions [36, 31]. In particular, Conan et al. [11] proposed an immersive setup to perform artistic 3D painting while dancing in which the symmetry is an essential part of the interaction to let the user dance in a artistic way without constraints from the system. In selection tasks, Ulinski et al. [50] performed evaluations that demonstrate a better accuracy with a symmetric interaction compared to two asymmetric ones. Authors explain this result due to the decreasing cognitive demand compared with an asymmetric interaction. Results show that the symmetric technique is the best if the time of the task is relatively short, else asymmetric techniques become better since they decrease arm strain issues.

Last, Balakrishnan et al. [5] showed that a poor design of a symmetric interaction technique can lead to a bimanual task performed in a sequential way. In this case, the interaction can be considered as asymmetric, and the performances of the bimanual task decrease due to the loss of parallel collaboration between both hands. Thus, symmetric interactions can be classified as a borderline case of asymmetric interactions.

To conclude, it appears that the choice between a symmetric or an asymmetric interaction technique is task-dependent. Indeed, according to the task, better interaction performances will be obtained in one setting or another. This choice must be guided by user studies that reveal the most appropriate technique depending on the required task.

\section{Collaborative interactions in CVE}

Extending bimanual interactions toward collaborative interactions involves difficulties linked to the increasing complexity of the interactions and to the coordination of potentially remote collaborators. First, subsection 5.1 focuses on interests to provide collaborative interaction techniques, then subsection 5.2 explains how some metaphors can easily be used in one setup or another without important modifications. Last, subsection 5.3 addresses specific difficulties implied by CVE and subsection 5.4 sums up this section

\subsection{Interests of collaborative interaction techniques}

Compared to uni-manual interactions, bimanual interactions have had a great success because they allow easier and more natural interactions in a lot of applications, for instance 3D manipulation [29] or virtual design [32] (cf. figure 2). In everyday life, there are still lots of interactions that are not wrapped into this class of interaction (e.g bimanual interactions). In particular, some tasks need multiple users acting at the same time on the same object to be achieved successfully. For example, some industrial manipulations [2, 44]

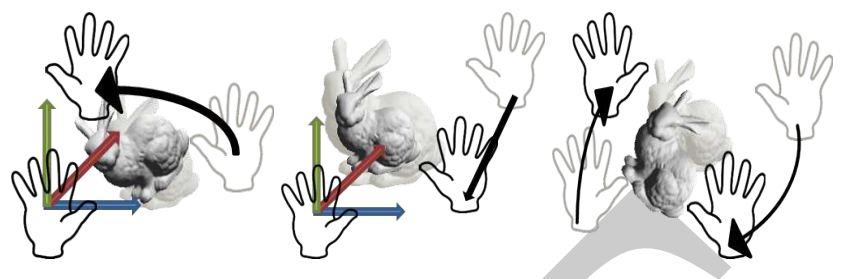

Figure 3: Illustrations of 3 manipulation techniques based on 2 6DOF inputs. Asymmetric: (1) trackball, (2) grab-and-scale. Symmetric: (3) slide-and-turn ( $\simeq$ grab-and-twirl)

cannot be done alone but need at least two collaborators to manipulate voluminous objects. In the same way, teaching scenarios involving a teacher and at least a learner [33] intrinsically require CVE. Moreover, other kinds of scenarios are performed better in a collaborative way. For instance, virtual exploration of large environment [38] can be enhanced by the help of a collaborator with a different point of view on the VE to guide the explorer.

\subsection{Collaborative interaction techniques as extensions from bimanual interaction techniques}

Most of the time, collaborative interactions use the split of DOF paradigm [41] exactly as bimanual asymmetric interactions. For example, several 3D manipulation techniques are asymmetric and can be used in both contexts such as the trackball or the grab-and-scale techniques. In the same way, symmetric interaction techniques such as the slide-and-turn or the grab-and-twirl are implemented in both bimanual and collaborative setups. Figure 3 illustrates these manipulation techniques. These kinds of interaction techniques based on multiple inputs share the same need for a controller. Controllers are the interfaces between several inputs and an interactive object. They generate merged outputs based on a specific policy. In this context, the controller's goal is to mediate the parallel and concurrent inputs in order to co-manipulate an object through multiple points of manipulation [3, 14]. This mediation depends on the implemented manipulation policy that can be based on a symmetric interaction paradigm (e.g average method) or on an asymmetric one (e.g split of DOF).

Controllers of these metaphors can always be reused or extended in a consistent way, and thus we note a strong parallel between bimanual and collaborative interactions: interaction metaphors are based on similar concepts of control. At this step, we could even reverse our approach noting that in fact a generic framework for (remote or local) collaboration could be used in bimanual scenarios seamlessly, e.g that bimanual is a specialization of collaboration for two local hands.

\subsection{Involved difficulties}

Comparisons between bimanual and collaborative interactions in asymmetric-based paradigm suffer from a strong limitation. Indeed, the role of proprioception in this bimanual interaction [4] is predominant and essential to successfully accomplish these kinds of complex tasks. However, in collaborative setups, collaborators cannot be aware of this internal sensory data. In order to overcome this limitation, additional feedbacks can be proposed to let the users perceive an external representation of this personal feedback. To achieve this, multiple sensory channels can be leveraged. Aguerreche et al. proposed a reconfigurable tangible device to be used in local co-manipulation tasks [2]. This device helps the users to feel the actions of others users by providing a direct and tangible link between the collaborators acting as a passive haptic interface. Sallnãs et al. used active haptic feedback that extends the feeling of other actions to distant collaborators [43]. In a similar way, vibrotactile [37] and pseudo-haptic [48] feedbacks can enhance interac- 
tions by providing direct data about others actions. All these sensory feedbacks (e.g haptic, visual and auditory) allow collaborators to have an external and potentially distant representation of other users actions who use their own internal proprioceptive sensory to act into the VE.

These direct feedbacks provide a representation of the distant users' activities. Thus, it helps collaborators coordination, especially in co-dependent interactions that request a strong synchronization of each user actions in a common task. It provides a collaborative aspect to the system interactivity, and is essential to let the shared VE be usable in a collaborative way.

\subsection{Summary}

We argued that bimanual and collaborative interactions follow the same goal that is to enhance interactions between a virtual world and one or several final users. Thus, we explained the similarities that can be found between the proposed interaction techniques in both fields. However, bimanual interactions are only a first step toward multiple input-based interactions. Collaboration is the next one and involves more complex and complete interaction techniques.

Bimanual interactions take into account only one user and are strongly based on the proprioceptive sensory of the user to enhance performances. In collaborative scenarios, the issue is to let collaborators perceive the other actions. This can be achieved through different feedback techniques that are wrapped in the term awareness. These awareness features are the difficult part to extend bimanual interactions toward collaborative interactions as explained in the next section.

\section{The aWAREnEsS ISSUE}

Awareness is essential to let the shared VE be usable in potentially distant collaborative tasks. It allows to perceive other actions and to understand the collaborators activities. Subsection 6.1 presents a classification of awareness features, then subsection 6.2 describes awareness solutions used to enhance the perception of the VE and its content. Subsection 6.3 develops some awareness techniques used in bimanual interaction techniques that could be reused in CVE. Last, subsection 6.4 describes some awareness solutions especially designed to enhance collaboration in CVE, and subsection 6.5 provides a final discussion.

\subsection{Awareness classification}

Classically in VR, systems' interactivity is defined as a 3-step loop that is: perception, decision, action. Another way to express it follows an interactive loop defined in three steps: awareness, action, feedback [26]. The first step ${ }^{2}$ indicates to the user what can be done in the VE, e.g what interactions are possible. The second step represents the actual interaction of the user with the VE, then the third step provides a feedback to let the user be aware of the consequences of his actions on the VE. Then, the loop can go on. Without it, there would be no mean for the user to directly understand his actions and the application would not be interactive enough. The lack of direct feedback drastically decreases the usability of the system. Goebbels et al. [26] proposed an extension of this interactive loop to be used in CVE. In particular, they extend the awareness part and divided it as follows:

1. Proprioception, e.g perception of his own actions.

2. Perception of his own physical and virtual input devices and of the virtual data set.

${ }^{2}$ The first step of the loop is named awareness in the literature. However, it only refers to the interaction capabilities awareness part of the whole awareness that the system can provide (that includes general feedbacks).
3. Perception of co-presence, e.g of other collaborators.

4. Perception of co-physical and virtual input devices and of covirtual data set (e.g shared data).

\section{Perception of co-knowledge and co-status.}

These five steps are the ordered steps perceived by users at each step of the awareness part of the interactive loop defined previously. We note that steps 1 and 2 are the same in a single-user or a multi-user setup, and steps 3 to 5 specifically address awareness features in collaborative settings. The term presence, in single-user settings, defines "the feeling of existence within a given environment" [51]. Sanchez et al. [45] claim that presence is a level of consciousness above awareness. Co-presence [26] extends this consciousness feeling for collaborators in shared worlds. The previous last 3 steps enable coordination between users and improve the use of collaborative interactions trying to compensate the lack of direct collaborators' proprioception feedback.

Awareness is not only studied by computer science researchers, it is inspired by work from the social science community, and adapted to the context of CSCW and CVE. Social science community provides an exhaustive classification of awareness, but CSCW and CVE do not require every features of this classification. A survey about similarities between awareness in CSCW and social sciences can be found in [27]. We rather present a specific classification of awareness for CVE taken from [24] and [40]. Thereby, awareness in CVE can be split into 6 classes:

1. Object: is it selectable? selected? who is its owner? can we modify some of its attributes?

2. Task: who currently participate in the task? what is the task status?

3. World: where are objects? users?

4. Group: who is online? what are the group members actions? positions? capabilities?

5. Social: what are the available communication channels?

6. System: what are the available data about the system states (stability, network latency,...)

This need of informations must be well-studied for each application and must not disturb the use of the system. For instance we must avoid to provide too many data that could hide some important parts of the VE. Rather, awareness must improve interaction performances by providing necessary visual, auditive or haptic cues together with a direct verbal communication channel at least. This must lead to enhance the whole perception of the VE including remote users, their current actions and their interaction capabilities.

\subsection{Awareness basics: VE and content}

First, VE must provide some ways to users to have an accurate perception of the whole environment. In immersive settings (e.g 1:1 scale), the World In Miniature (WIM) technique provides a way to have a global view of the whole VE in a map-like view. Without it, users only see what is contained in their respective view frustum. Most of the time in non-immersive setups, the available horizontal field of view (fov) is limited to $60^{\circ}$, whereas natural range of binocular vision extends to $120^{\circ}$, and up to $200^{\circ}$ in monocular vision. Fraser et al. [20] proposed peripheral lenses to provide such a wide horizontal fov. These peripheral lenses are placed on both sides of the central classical $60^{\circ}$ fov and display another $60^{\circ}$ fov on a thinner width. It provides peripheral blurred views mimicking natural peripheral vision. Users can momentarily switch the clear view and 


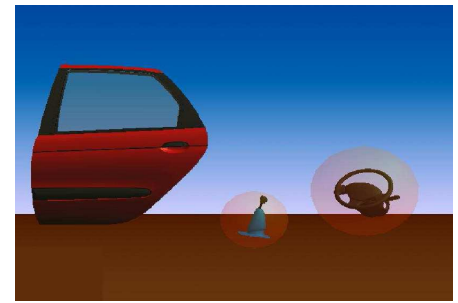

(a)

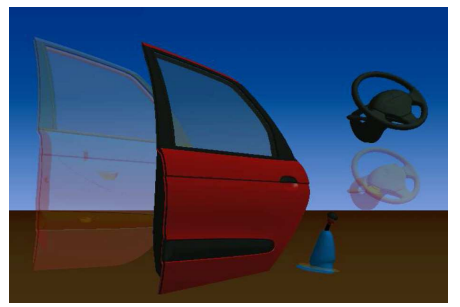

(b)

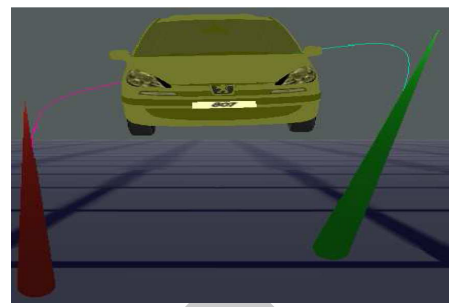

(c)

Figure 4: Illustrations of visual cues used to enhance awareness in CVE: (a) Bounding boxes indicating a specific object property [16], (b) Echoes managing network troubles [16], (c) Rays from the owners to the manipulated object [1].

thus better perceive their environment and the collaborators' location and action.

Second, VE can let users be aware of the objects' interaction capabilities to improve their usability. For instance, interactive objects can display their current state by changing some of its appearance attributes when they are selected or manipulated. Another way to represent it is to display a semi-transparent bounding-box (cf. figure 4a. Note that this method can also be extended using echoes in order to inform users about network troubles concerning shared virtual objects [16] (cf. figure 4b). Moreover, when an object is selected or manipulated by a user named an owner, it is interesting for other users to know who is this owner. Typical technique consists in displaying the owner's name next to the interactive object, but more visual awareness techniques propose to display a ray (or an extended arm) from the owner location to the object (cf. figure 4c). Concerning interaction capabilities, objects can provide visual cues such as manipulation arrows in order to inform the users which interactions are possible.

Last, a famous approach considered two distinct spaces for each user named the focus and the nimbus [8]. In [7], focus and nimbus are "subspaces within which an object chooses to direct either its presence or its attention". More specifically, "the more an object is within your focus, the more aware you are of it", and "the more an object is within your nimbus, the more aware it is of you". It means that, in a generic way, interactive objects can react to user position and focus by changing appearances for example, and also inform others about user's attention and interaction capabilities.

\subsection{Bimanual awareness solutions}

In single-user setup, awareness is a key feature too. Particularly in bimanual interactions, the system can provide awareness through three different channels. First, visual feedback of the interactive tool allows the user to better anticipate the consequences of his actions. It is essential in immersive setups as in [32] to improve the immersive feeling of the user in the VE. Second, auditory feedback can enhance user perception of the VE. For instance, physical behaviors can be simulated thanks to specific sounds, such as contacts and friction in [32]. Third, haptic feedback improves kinaesthetic feedback and helps the user to coordinate his movements. In [36], Murayama et al. used two SPIDAR devices, one for each hand. They provided separate haptic feedback for each hand and increased the bimanual interaction abilities thanks to the feeling of forces applied by hands in the VE. Other pseudo-haptic feedbacks such as proposed in [48] can provide visual or auditory cues to overcome the lack of haptic device.

\subsection{Awareness techniques for CVE}

In the literature, many solutions have been implemented to enhance collaboration in CVE thanks to awareness. We do not present an exhaustive list of these solutions, rather we discuss some of them that we think they are widely used and generic enough.

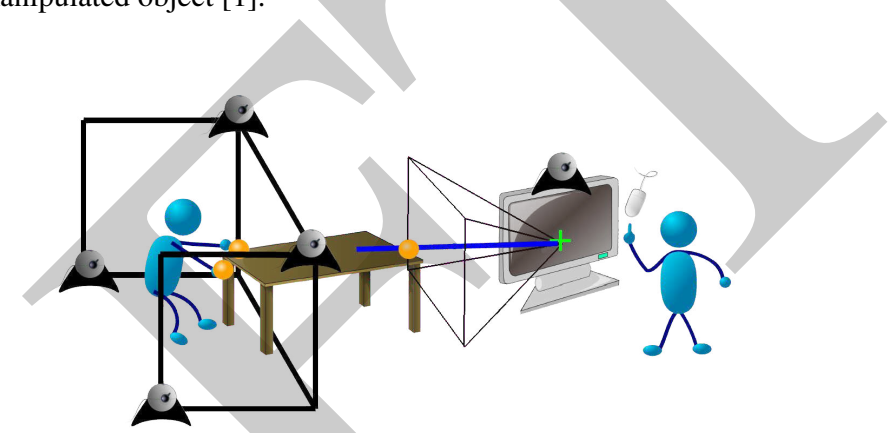

Figure 5: An illustration of a collaborative session with two users. The one on the left works in an immersive setup, while the one on the right uses a desktop setup [15].

\subsubsection{Awareness of collaborators}

First, users have to be able to perceive their collaborators into the shared VE. To achieve it, the typical solution is to use an avatar animated according to the user motion. In the simplest case, only the body of the avatar is animated, but more advanced research worked on realistic avatar [6] textured as the real user, and even emotive avateering [21] in order to enhance non-verbal communication between remote users. In this field, an interesting modality is gaze tracking that enables eye-contact and awareness of others' gaze [49]. Avatars with gaze direction allow fine and informative non-verbal communication and can reproduce natural social interactions.

Second, users should perceive others' interactions capabilities to be able to collaborate effectively. These capabilities can depend on hardware setups because users can interact with heterogeneous setups, such as an immersive visualization room with full body tracking, a semi-immersive 3D display with a 6DOF tracker input or a head-mounted display with tracked hands. Figure 5 gives an illustration of two heterogeneous setups. This heterogeneity leads to different interaction capabilities for each user. Collaboration becomes more effective if everyone is aware of these limitations. Duval et al. [17] proposed the Immersive and Interactive Virtual Cabin (IIVC) as a generic concept to handle this awareness feature. It allows to define the real 3D volumes that represent different workspaces linked to a specific modality (e.g motion, visual, auditory,...) and to let other users perceive these spaces. These virtual spaces can be linked to some tracking limitation or screen size, and to be aware of that avoid misunderstanding between collaborators, for example representing the view frustum [20]. Figure 6 explicates the generic concept of the IIVC.

\subsubsection{Collaborative awareness through communication}

In this paragraph, we present general awareness techniques providing ways to users to communicate. First, CVE must allow direct and 


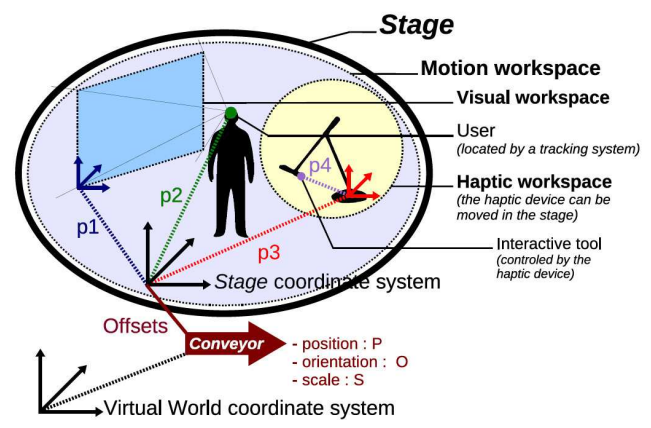

Figure 6: Explicative scheme of the different workspaces composing the IIVC and their relations [17].

natural communication. Several channels can be employed such as an audio channel. The audio communication has been proved to be the most important and essential for effective collaboration. Video [39] or haptic [10] channels can also be used. Second, symbolic communication is another way to allow communication between remote users. For instance, users can deposit arrows, compass or lighting into the VE to inform collaborators about interesting spots [38]. They can also create notes and post-it to add informations they collect in the VE and to share it with collaborators.

\subsection{Discussion}

Awareness techniques used in systems based on bimanual interactions providing visual feedback of the interactive tools, as auditory and haptic feedback, can be used in collaborative setups too. In this context, they allow remote users to perceive interactive tools and actions of other users[39]. In the same way, it enhances coordination, especially in co-manipulation tasks through visual [24], auditory and haptic/tactile [37] cues. Experiments proved that these awareness techniques improve task performances in collaborative settings too.

Table 7 summarizes main aspects of similarities that can be found between the two fields we are interested in, and highlights some of the CVE challenges. These challenges are mainly due to the remote aspect and the loss of proprioceptive feedback compared with bimanual interactions.

Bimanual

Symmetric

Interaction metaphors Asymmetric

Control interfaces (controllers)

\begin{tabular}{|c|c|}
\hline \multirow{4}{*}{ Awareness techniques } & VE and contents capabilities perception \\
\cline { 2 - 2 } & $\begin{array}{r}\text { Feedbacks of interactions with the system } \\
\text { Tools representation }\end{array}$ \\
\cline { 2 - 2 } & $\begin{array}{c}\text { Collaborators perception } \\
\text { (capabilities, activities,...) }\end{array}$ \\
\cline { 2 - 2 } Communication channels \\
\hline
\end{tabular}

Figure 7: Synthesis table illustrating the similarities between bimanual and collaborative interaction techniques. Some features have an equivalent in both fields, others are shared, and last, some are specific to CVE.

\section{Conclusion and Perspectives}

This preliminary study shows that multiple input-based metaphors used for bimanual interactions can partially inspire the design of collaborative interaction techniques. However, even if bimanual interactions bring large improvements compared to uni-manual interactions, collaborative interactions are yet another step beyond. Thus, they imply more complexity and have to be specifically designed for this purpose. However, some guidelines can be picked from the existing bimanual interaction techniques such as the choice between symmetric or asymmetric collaboration according to the task. As well, some awareness issues have been handled by bimanual interaction techniques such as the tools representation or the action explicitness by different means (visual, haptic, auditive,...) and can be re-employed in CVE. However, this awareness aspect is more important in CVE and then must propose new techniques to let the users have an accurate perception of the shared VE including remote users. An important feature improving coordination is the availability to perceive distant activities to be able to anticipate collaborators' actions. Thus, co-dependent interactions can take place in a comprehensive way. This collaborative awareness aspect will drive our future work and we will try to propose innovative solutions to let remote users have a perception of the collaborators' activity through specific feedbacks based on immersion, awareness and presence.

\section{REFERENCES}

[1] L. Aguerreche, T. Duval, B. Arnaldi, et al. A description of a dia$\log$ to enable interaction between interaction tools and 3D objects in collaborative virtual environments. VRIC 2009, page 63-73, 2009.

[2] L. Aguerreche, T. Duval, and A. Lécuyer. Reconfigurable tangible devices for 3D virtual object manipulation by single or multiple users. In Proceedings of the 17th ACM Symposium on Virtual Reality Software and Technology, page 227-230, 2010.

[3] L. Aguerreche, T. Duval, A. Lécuyer, et al. Short paper: 3-hand manipulation of virtual objects. JVRC 2009, 2009.

[4] R. Balakrishnan and K. Hinckley. The role of kinesthetic reference frames in two-handed input performance. In Proceedings of the 12th annual ACM symposium on User interface software and technology, page 171-178, 1999.

[5] R. Balakrishnan and K. Hinckley. Symmetric bimanual interaction. In Proceedings of the SIGCHI conference on Human factors in computing systems, page 33-40, 2000.

[6] S. Beck, A. Kunert, A. Kulik, and B. Froehlich. Immersive groupto-group telepresence. Visualization and Computer Graphics, IEEE Transactions on, 19(4):616-625, 2013.

[7] S. Benford, J. Bowers, L. E. Fahlén, and C. Greenhalgh. Managing mutual awareness in collaborative virtual environments. In Proceedings of the Conference on Virtual Reality Software and Technology, VRST '94, page 223-236, River Edge, NJ, USA, 1994. World Scientific Publishing Co., Inc.

[8] S. Benford and L. E. Fahlén. Awareness, focus, and aura: A spatial model of interaction in virtual worlds. In HCI (2), pages 693-698, 1993.

[9] F. Bérard. The magic table: Computer-vision based augmentation of a whiteboard for creative meetings. IEEE Workshop on Projector Camera Systems, 2003.

[10] A. Chellali, C. Dumas, and I. Milleville. Haptic communication to enhance collaboration in virtual environments. In Proceedings of the 28th Annual European Conference on Cognitive Ergonomics, page 83-90. ACM, 2010.

[11] A. Clay, J.-C. Lombardo, N. Couture, J. Conan, et al. Bi-manual 3D painting: an interaction paradigm for augmented reality live performance. In HCITOCH-Human-Computer Interaction, Tourism and Cultural Heritage-2012, 2012

[12] F. Coldefy and S. Louis-dit Picard. DigiTable: an interactive multiuser table for collocated and remote collaboration enabling remote gesture visualization. In Computer Vision and Pattern Recognition, 2007. CVPR'07. IEEE Conference on, page 1-8, 2007.

[13] L. D. Cutler, B. Fröhlich, and P. Hanrahan. Two-handed direct manipulation on the responsive workbench. In Proceedings of the 1997 symposium on Interactive 3D graphics, page 107-114, 1997. 
[14] T. Duval, A. Lécuyer, and S. Thomas. Skewer: a 3d interaction technique for 2-user collaborative manipulation of objects in virtual environments. In 3D User Interfaces, 2006. 3DUI 2006. IEEE Symposium on, page 69-72, 2006.

[15] T. Duval, T. T. H. Nguyen, C. Fleury, A. Chauffaut, G. Dumont, and V. Gouranton. Improving awareness for 3D virtual collaboration by embedding the features of users' physical environments and by augmenting interaction tools with cognitive feedback cues. Journal on Multimodal User Interfaces, 2013.

[16] T. Duval, C. Zammar, et al. Managing network troubles while interacting within collaborative virtual environments. In CSAC, page 85-94, 2006

[17] C. Fleury, A. Chauffaut, T. Duval, V. Gouranton, and B. Arnaldi. A generic model for embedding users' physical workspaces into multiscale collaborative virtual environments. In ICAT 2010 (20th International Conference on Artificial Reality and Telexistence), 2010.

[18] C. Fleury, T. Duval, V. Gouranton, and B. Arnaldi. Architectures and mechanisms to efficiently maintain consistency in collaborative virtual environments. In Proc. of Software Engineering and Architectures for Realtime Interactive Systems - SEARIS, page 87-94, 2010.

[19] C. Fleury, T. Duval, V. Gouranton, and A. Steed. Evaluation of remote collaborative manipulation for scientific data analysis. In Proceedings of the 18th ACM symposium on Virtual reality software and technology, page 129-136, 2012.

[20] M. Fraser, S. Benford, J. Hindmarsh, and C. Heath. Supporting awareness and interaction through collaborative virtual interfaces. In Proceedings of the 12th annual ACM symposium on User interface software and technology, page 27-36, 1999.

[21] D. Freeman, M. Slater, P. E. Bebbington, P. A. Garety, E. Kuipers, D. Fowler, A. Met, C. M. Read, J. Jordan, and V. Vinayagamoorthy. Can virtual reality be used to investigate persecutory ideation? The Journal of nervous and mental disease, 191(8):509-514, 2003.

[22] B. Frohlich, J. Plate, J. Wind, G. Wesche, and M. Gobel. Cubicmouse-based interaction in virtual environments. Computer Graphics and Applications, IEEE, 20(4):12-15, 2000.

[23] P. Fuchs, G. Moreau, and P. Guitton. Virtual reality: concepts and technologies. CRC Press, Inc., 2011.

[24] A. S. García, J. P. Molina, D. Martínez, and P. González. Enhancing collaborative manipulation through the use of feedback and awareness in CVEs. In Proceedings of The 7th ACM SIGGRAPH International Conference on Virtual-Reality Continuum and Its Applications in Industry, page 32, 2008.

[25] P. Gaucher, F. Argelaguet, J. Royan, and A. Lécuyer. A novel 3D carousel based on pseudo-haptic feedback and gestural interaction for virtual showcasing. In 3D User Interfaces (3DUI), 2013 IEEE Symposium on, page 55-58, 2013.

[26] G. Goebbels, V. Lalioti, and M. Göbel. Design and evaluation of team work in distributed collaborative virtual environments. In Proceedings of the ACM symposium on Virtual reality software and technology, page 231-238, 2003.

[27] T. Gross, C. Stary, and A. Totter. User-centered awareness in computer-supported cooperative work-systems: Structured embedding of findings from social sciences. International Journal of Human-Computer Interaction, 18(3):323-360, 2005.

[28] Y. Guiard and T. Ferrand. Asymmetry in bimanual skills. Manиal asymmetries in motor performance, page 175-195, 1996.

[29] K. Hinckley, R. Pausch, D. Proffitt, and N. F. Kassell. Two-handed virtual manipulation. ACM Transactions on Computer-Human Interaction (TOCHI), 5(3):260-302, 1998.

[30] C. Joslin, T. Di Giacomo, and N. Magnenat-Thalmann. Collaborative virtual environments: from birth to standardization. Communications Magazine, IEEE, 42(4):28-33, 2004.

[31] C. Latulipe, S. Mann, C. S. Kaplan, and C. L. Clarke. symSpline: symmetric two-handed spline manipulation. In Proceedings of the SIGCHI conference on Human Factors in computing systems, page 349-358, 2006.

[32] M. Le Chénéchal, B. Arnaldi, and M. Marchal. Poster: Bimanual design of deformable objects thanks to the multi-tool visual metaphor. In $3 D$ User Interfaces, 2014.

[33] A. S. Luna, V. Gouranton, and B. Arnaldi. Collaborative virtual en- vironments for training: A unified interaction model for real humans and virtual humans. In E-Learning and Games for Training, Education, Health and Sports, page 1-12. Springer, 2012.

[34] D. Margery, B. Arnaldi, and N. Plouzeau. A general framework for cooperative manipulation in virtual environments. In Virtual Environments' 99, page 169-178. Springer, 1999.

[35] J. D. Mulder and B. Boscker. A modular system for collaborative desktop vr/ar with a shared workspace. In Virtual Reality, 2004. Proceedings. IEEE, page 75-280, 2004

[36] J. Murayama, L. Bougrila, Y. Luo, K. Akahane, S. Hasegawa, B. Hirsbrunner, and M. Sato. SPIDAR G\&G: a two-handed haptic interface for bimanual VR interaction. In Proceedings of EuroHaptics, page 138-146, 2004.

[37] M. Naud, S. Ullah, P. Richard, S. Otmane, M. Mallem, et al. Effect of tactile feedback and viewpoint on task performance in a collaborative virtual environmment. In Joint virtual reality conf of EGVE-ICATEuroVR, 2009.

[38] T. T. H. Nguyen, T. Duval, C. Fleury, et al. Guiding techniques for collaborative exploration in multi-scale shared virtual environments. In Proceedings of GRAPP International Conference on Computer Graphics Theory and Applications, 2013.

[39] A. Pauchet, F. Coldefy, L. Lefebvre, S. L. D. Picard, A. Bouguet, L. Perron, J. Guerin, D. Corvaisier, and M. Collobert. Mutual awareness in collocated and distant collaborative tasks using shared interfaces. In Human-Computer Interaction-INTERACT 2007, page 59-73. Springer, 2007.

[40] M. S. Pinho, D. A. Bowman, and C. M. Freitas. Cooperative object manipulation in immersive virtual environments: framework and techniques. In Proceedings of the ACM symposium on Virtual reality software and technology, page 171-178, 2002.

[41] M. S. Pinho, D. A. Bowman, and C. M. D. S. Freitas. Cooperative object manipulation in collaborative virtual environments. Journal of the Brazilian Computer Society, 14(2):53-67, 2008.

[42] J. Rekimoto. Transvision: A hand-held augmented reality system for collaborative design. In Proc. Virtual Systems and Multimedia, page 85-90, 1996.

[43] E.-L. Sallnäs, K. Rassmus-Gröhn, and C. Sjöström. Supporting presence in collaborative environments by haptic force feedback. ACM Transactions on Computer-Human Interaction (TOCHI), 7(4):461-476, 2000.

[44] H. Salzmann, J. Jacobs, and B. Froehlich. Collaborative interaction in co-located two-user scenarios. In JVRC 2009 (Joint Virtual Reality Conference of EGVE - ICAT - EuroVR 2009), page 85-92, 2009.

[45] M. V. Sanchez-Vives and M. Slater. From presence towards consciousness. In 8th Annual Conference for the Scientific Study of Consciousness, 2004.

[46] A. Simon and S. Scholz. Multi-viewpoint images for multi-user interaction. In Virtual Reality, 2005. Proceedings. VR 2005. IEEE, page 107-113. IEEE, 2005.

[47] D. Snowdon, E. F. Churchill, and A. J. Munro. Collaborative virtual environments: Digital spaces and places for CSCW: an introduction. In Collaborative Virtual Environments, page 3-17, 2001.

[48] J. Sreng, A. Lecuyer, and C. Megard. Using visual cues of contact to improve interactive manipulation of virtual objects in industrial Assembly/Maintenance simulations. Visualization and Computer Graphics, 12:1013-1020, 2006.

[49] W. Steptoe, R. Wolff, A. Murgia, E. Guimaraes, J. Rae, P. Sharkey, D. Roberts, and A. Steed. Eye-tracking for avatar eye-gaze and interactional analysis in immersive collaborative virtual environments. In Proceedings of the 2008 ACM conference on Computer supported cooperative work, page 197-200, 2008.

[50] A. Ulinski, C. Zanbaka, Z. Wartell, P. Goolkasian, and L. F. Hodges. Two handed selection techniques for volumetric data. In $3 D$ User Interfaces, 2007. 3DUI'07. IEEE Symposium on, 2007.

[51] P. Zahorik and R. L. Jenison. Presence as being-in-the-world. Presence: Teleoperators and virtual environments, 7(1):78-89, 1998.

[52] T. G. Zimmerman, J. Lanier, C. Blanchard, S. Bryson, and Y. Harvill. A hand gesture interface device. In ACM SIGCHI Bulletin, volume 18, page 189-192. ACM, 1987. 